\title{
Aharonov-Bohm signature for neutral excitons in type-II quantum dot ensembles
}

\author{
E. Ribeiro* G. Medeiros-Ribeiro, and W. Carvalho Jr. \\ Laboratório Nacional de Luz Síncrotron, \\ PO Box 6192, 13084-971 Campinas - SP, Brazil \\ A. O. Govorov \\ Ohio University, Department of Physics and Astronomy, \\ Clippinger Research Labs, Athens $\mathrm{OH}$ 45701, USA
}

(Dated: August 26, 2018)

\begin{abstract}
The Aharonov-Bohm (AB) effect is commonly believed to be a typical feature of the motion of a charged particle interacting with the electromagnetic vector potential. Here we present a magnetophotoluminescence study of type-II InP/GaAs self-assembled quantum dots, revealing the Aharonov-Bohm-type oscillations for neutral excitons when the hole ground state changes its angular momentum from $\ell_{h}=0$ to $\ell_{h}=1,2$, and 3 . The hole ring parameters derived from a simple model are in excellent agreement with the structural parameters for this system.
\end{abstract}


In 1959, Aharonov and Bohm proposed an experiment to assess the manifestations of the electromagnetic potentials in the quantum domain [1]. By allowing a charged particle to circulate a confined magnetic field flux region, they showed that surprisingly, there exist effects of the vector potential on the charged particles moving outside. After the circulation the particle wavefunction acquires a phase that is proportional to the magnetic flux contained within the closed path [2]. Therefore, all observable phenomena depend upon the flux $\Phi$ through the excluded region, and are shown to be periodic with period $\left.\Phi_{0}=h c / e\right] 3$, 4,, 5$]$. This oscillatory characteristic is the signature of the Aharonov-Bohm (AB) effect [1, 6].

The definite measurement of the original $\mathrm{AB}$ effect was performed by Tonomura et al. in a very clever experiment $[6,7]$. Investigation and applications of the $A B$ concepts in condensed matter have been carried out in metals [8] and superconductors [3, 4, [9]. With the advance of lithography and semiconductor growth techniques, it was possible to devise mesoscopic 10 , 11] and nanoscopic [12,13] devices where many theoretically predicted signatures evidencing ring-like electronic properties [14, 15, 16] have been observed by transport experiments. For the case of few electrons, self-assembled quantum rings [17] were used for the observation of the characteristic spectra including excitations of states of different angular momentum 17]. Due to the small sizes of these structures, it was possible for the carriers to maintain the coherence of their wavefunctions during their motion (at sufficiently low temperatures), thus allowing the observation of relative quantum phase effects (AB-like).

It was proposed that a neutral excitation could also exhibit such an AB oscillatory behavior [18, 19]. Electron-hole pairs created by optical excitation and bound together via Coulomb interaction (excitons) are a good candidate when either one or both carriers are confined to a ring-like geometry. The existence of the $\mathrm{AB}$ oscillations for neutral excitons in semiconductor quantum rings, where both carriers are confined in the rings, has been a matter of controversy in recent years 18, 20, 21, 22, 23, 24] since some of the work predicted that the $\mathrm{AB}$ oscillations might be very weak because of exponentially small electron-to-hole tunnel amplitude [18, 19] or would vanish for a finite width ring [21, 22]. Recently, the AB effect was observed in optical experiments by Bayer et al. on quantum-rings fabricated by lithographical methods [25]. They recorded AB oscillations for charged excitons. Perhaps due to the aforementioned reasons, the $\mathrm{AB}$ effect was not found in the spectra of neutral excitons. The second possibility for observing AB-like oscillations in the optical spectra of a neutral excitation is the case of type-II quantum dots (QD) [19, 23, 26, 27], where 
the confinement of one carrier inside the QD and the other carrier in the barrier naturally creates a ring-like structure (see Fig. 1). In such a structure, the carrier outside the QD would be bound to that confined in the QD via Coulomb attraction. This spatial charge separation produces a polarization of the exciton and thus $\mathrm{AB}$ oscillations could be observed in the energy of those carriers that are confined in the ring-shaped orbit around the QD. Moreover, the $\mathrm{AB}$ effect with a polarized exciton may not involve an electron-to-hole tunnel amplitude [19, 23]. There are several self-assembled quantum dot systems where a type-II heterojunction is expected; for instance, GaSb on GaAs 28] and Ge on Si [29] provide a three-dimensional confinement for the holes, thereby creating a ring-like potential for the electrons. Alternatively, InP on InGaP [30] and InP on GaAs 31] provide the localization of the electrons in the QD, and consequently a ring-like hole wavefunction. Figure 1 sketches the valence band potential in a very simplified way, not taking into account the contribution of the strain. It is known that the QD strains the GaAs substrate and cap layer, and that in turn changes the potential landscape in the GaAs, creating an additional ring-like potential around the QD for heavy-hole confinement [32]. The light hole may have a well-like strain-induced potential below the QD [32], however being shallower than the heavy hole ring potential. InP QDs are particularly suitable for observing oscillatory behavior with $\mathrm{AB}$ period $\Phi_{0}$. With diameters of the order of $35 \mathrm{~nm}$ one obtains a period of few Tesla for the $\mathrm{AB}$ effect.

Here we present data on InP/GaAs self-assembled quantum dot ensembles where the confined magnetoexcitons trap fluxes in the range of 0-3 flux quanta, corresponding to a magnetic field span of 0-12 T. Samples were grown at different rates and $\mathrm{PH}_{3}$ fluxes by metal-organic chemical vapor deposition in a commercial reactor on nominally flat semiinsulating Cr-doped GaAs [001] substrates at $550{ }^{\circ} \mathrm{C}$ [31]. The structures consisted of a $300 \mathrm{~nm}$ undoped GaAs grown at $600{ }^{\circ} \mathrm{C}$ buffer layer followed by the QD layer. The islands were then capped with a $50 \mathrm{~nm}$ undoped GaAs cap. For this work, the sample with the narrowest luminescence line width $(\sim 35 \mathrm{meV})$ was chosen, representing an ensemble of $3 \times 10^{10} \mathrm{QDs} / \mathrm{cm}^{2}$ [31] with small size dispersion. The dimensions of the QDs were determined by cross-section transmission electron microscopy (TEM) [33], and were found to be $(32 \pm 6) \mathrm{nm}$ and $(4 \pm 2) \mathrm{nm}$ in average diameter and height. The InP QDs are not cylindrically symmetric, being elongated in the [110] direction. The photoluminescence (PL) experiments were performed at $2 \mathrm{~K}$, with magnetic fields up to $12 \mathrm{~T}$ (in $0.2 \mathrm{~T}$ steps), 


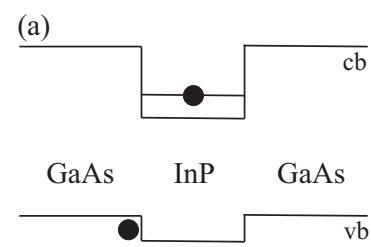

(b)

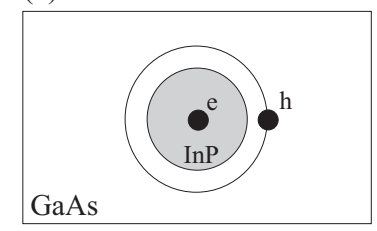

FIG. 1: Sketches of the type-II InP/GaAs QDs: (a) conduction and valence band profiles, indicating the spatial separation of electrons and holes; (b) top view of the QD plane, indicating the holes confined to a ring around the QD due to the Coulomb interaction with the electron trapped into the dot.

and using an Ar laser with $2 \mathrm{~W} / \mathrm{cm}^{2}$ as excitation source. These conditions guaranteed the filling of only the system ground state, allowing the assessment of single particle energies. For higher excitation power a line broadening and luminescence shift [34] takes place, indicating excited state population, which would complicate our analysis.

Fig. 2 shows the InP QDs PL spectra for $B=0$ (circles) and $B=12 \mathrm{~T}$ (triangles). The total energy shift within this field range was $4.5 \mathrm{meV}$. The PL peak intensity increased about $50 \%$ from 0 to $12 \mathrm{~T}$, indicating an enhancement of the electron and hole wavefunction overlap. The spectra for all the field values were fit with a single Gaussian line shape in order to extract the evolution of the PL peak position of the InP QDs as a function of the applied magnetic field, $E_{P L}(B)$, which is plotted in Fig. 3(a). There one can see that instead of the typical monotonic diamagnetic energy shift characteristics of type-I QD [35], the InP QD PL shows an oscillatory behavior in the $E_{P L}(B)$ curve, very similar to what has been predicted by Kalameítsev and collaborators [19]. In that work, it was expected that the maxima of oscillating part in $E_{P L}(B)$ would develop every time that the lower-lying carrier state changed its angular momentum from $\ell_{e}=0$ to $\ell_{e}=-1$ and so forth. These transitions were seen to occur when $R^{2} / l_{B}^{2} \sim\left|\ell_{e}\right|[19]$, with $R$ being the ring radius, $l_{B}^{2}=\hbar / e B$ the magnetic length and $\ell_{e}$ the electron state angular momentum. Rewriting this expression for the holes in InP QDs, $R \sim\left(\hbar \ell_{h} / e B\right)^{1 / 2}$ and then by inserting the experimental values of $B$ 


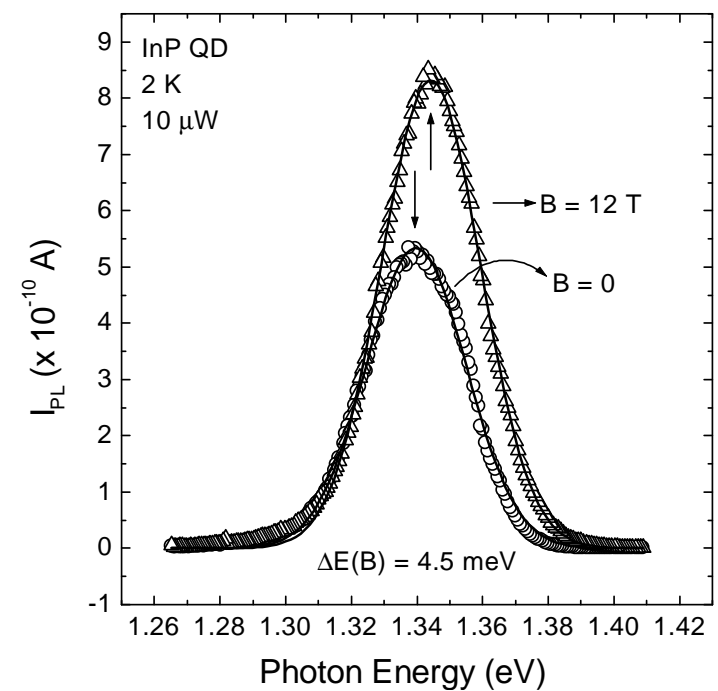

FIG. 2: PL spectra of the InP QDs for $B=0$ (circles) and $B=12 \mathrm{~T}$ (triangles). Solid lines are Gaussian fits to the data. The total energy shift is $4.5 \mathrm{meV}$ for $12 \mathrm{~T}$.

for the observed plateau-like regions [Figure 3(a)] an estimate for the hole ring radius can be obtained. Averaging the results for the three plateaux, a radius of $R=(16 \pm 1) \mathrm{nm}$ is obtained, in agreement to what has been measured by TEM [33].

Nevertheless, it is possible to further elaborate the analysis of the data. The electron ground state of InP/GaAs QDs has been investigated by capacitance spectroscopy (CV) [36]. It has already been established [35] that the lateral confinement for the self-assembled quantum dot can be reasonably modelled by a parabolic potential. The diamagnetic shift of the electron ground state can be described by $E_{e}=\left(\left(\hbar \omega_{0}\right)^{2}+\left(\hbar \omega_{c}\right)^{2} / 4\right)^{1 / 2}$ [37], with $\omega_{c}$ being the cyclotron frequency. For InP/GaAs QDs grown under identical nominal conditions, a characteristic energy of $\hbar \omega_{0}=(5.6 \pm 0.9) \mathrm{meV}$ was inferred [36]. Bearing this information in mind, an expression for $E_{P L}(B)$ can be written, consisting of three terms: a constant contribution, related to the energy gap of the material and the exciton binding energy; the diamagnetic energy shift for the electrons, discussed above; and the variation of the hole energy with the magnetic field [24, 26]:

$$
E_{P L}(R, B)=E_{g}+\sqrt{\left(\hbar \omega_{0}\right)^{2}+\left(\frac{\hbar \omega_{c}}{2}\right)^{2}}+\frac{\hbar^{2}}{2 m_{h}^{*} R^{2}}\left(\ell_{h}-\frac{\Phi}{\Phi_{0}}\right)^{2},
$$

where $m_{h}^{*}$ is the hole effective mass, $\Phi_{0}=h c / e$ is the flux quantum and $\Phi=\pi R^{2} B$ is the total magnetic flux through the ring of radius $R$. Using the above result for $\hbar \omega_{0}$, both 
the constant and the electron contributions in the experimental data [see solid curve in Fig. 3(a)] can be subtracted. The result is shown in Fig. 3(b), where:

$$
E_{h}(R, B)=E_{P L}(R, B)-E_{g}-E_{e}=\frac{\hbar^{2}}{2 m_{h}^{*} R^{2}}\left(\ell_{h}-\frac{\Phi}{\Phi_{0}}\right)^{2} .
$$

As shown in Fig 3(b), $E_{h}$ (open squares) clearly oscillates as a function of the magnetic field, indicating phase coherence for the hole wavefunctions. Since $\Phi_{0}$ is a constant (the Aharonov-Bohm period of the excitonic energy oscillations [1, 6, 15]), the experimental data were fit with the parabolas given by Eq. (2), one for each value of $\ell_{h}=0,1,2$, and 3 , indicating the number of flux quanta enclosed by the hole ring. The result is shown by the dashed curves in the upper part of Fig. 3(b), from which $R=(19.1 \pm 0.4) \mathrm{nm}$ and $\Phi_{0}=3.61$ $\mathrm{T}$ were obtained. One should keep in mind that the hole ring must have a radius slightly larger than the QD diameter (Fig. 1), and therefore the obtained values are in excellent agreement with the structural and electronic results discussed above [33].

It is worth noting that an ensemble of islands and not a single QD was probed. Nevertheless, the size dispersion did not suppress the AB oscillations. Some considerations can be done on the influence of the size dispersion $\Delta R$ on the experimental results. From CV experiments [36] an effective size distribution of $\Delta R_{C V}=0.8 \mathrm{~nm}$ was inferred for the electron-confining potential. This $\mathrm{CV}$ estimate for $\Delta R$ would be a lower bound for the experimentally-probed size dispersion. In order to model in a more quantitative fashion the effect of the QD ensemble on the hole-ring energy oscillations, $E_{h}(R, B)$ [eq. (2)] was calculated and weighted by a Gaussian size distribution function, where $R$ determined in Fig. $3(\mathrm{~b})$ is the central peak radius and $2 \Delta R_{C V}$ is its full width at half maximum. The result is plotted in Fig. 3(b) (solid curve). It can be seen that the main effect of the size dispersion on $E_{h}$ is a progressive attenuation of the oscillation intensity, as one would expect, and it is consistent with the decrease in the amplitude of the data in Fig. 3(b). By comparing the data and the solid curve in Fig. 3(b) one can see that the experimental data of the hole ring are consistent with an effective size dispersion obtained from $\Delta R_{C V}$.

Although describing the oscillation period and damping in a good agreement with independent structural/electronic results, this simplified model fails to predict the energy offset of the last parabola (corresponding to $\ell=3$ ). The contributions for the offset might be: (i) the experimental uncertainty in $\hbar \omega_{0}$ obtained from the CV fit [solid curve in Fig 3(a)]; (ii) a possible dependence of the exciton binding energy on the magnetic field; (iii) devia- 


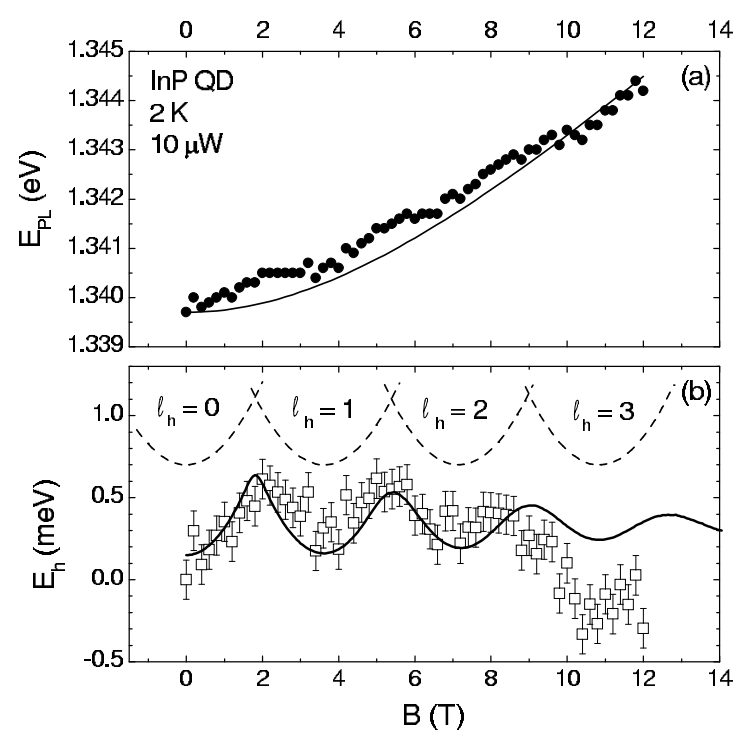

FIG. 3: (a) The PL peak position as a function of the applied magnetic field (solid circles). Three plateau-like structures are clearly observed. The error bars are smaller than the solid circles. (b) Hole energy dependence on the magnetic field (open squares), showing the Aharonov-Bohm oscillations with period $\phi_{0}$. The error bars are the uncertainties on the peak position after the Gaussian fits. The dashed curves are parabolas following Eq. (2) shifted upward by arbitrary energy. Calculated $E_{h}$ [Eq. (2)] for a QD ensemble described by a Gaussian size distribution for $R$, with $R_{\text {peak }}=19.1 \mathrm{~nm}$ and full width at half maximum of $2 \Delta R_{C V}=1.6 \mathrm{~nm}$ is shown by the solid curve. Note the progressive offsets of the parabola minima for increasing $\ell_{h}$.

tions from the parabolic lateral confinement due to the actual QD morphology. Clearly, a more detailed and complete modelling of the system, including the points indicated above, is needed for understanding all the experimental features presented in Fig. 3(b), but such a task is beyond the scope of the present work. Nevertheless, the simple model used here consistently describes the main physical issues connected to the observation of the hole AB oscillations (Fig. 3).

In summary, we have presented clear evidence of Aharonov-Bohm oscillations in the spectrum of electrically neutral excitons confined in type-II InP/GaAs quantum dots. We have observed the trapping of up to three flux quanta inside the ring trajectory of the hole, for a reasonable span of magnetic fields. Despite the presence of a size dispersion, the $\mathrm{AB}$ oscillations were easily observable, evidencing the phase coherence for holes moving around QDs. 
The magnetic field experiments were performed at the Optical Properties Group facilities at Campinas State University (Unicamp). We would like to thank H. Gazetta Filho for the technical support on sample growth. We gratefully acknowledge financial support from Fundação de Amparo à Pesquisa do Estado de São Paulo and MCT-CNPq.

* Electronic address: evaldo@lnls.br

[1] Y. Aharonov and D. Bohm, Phys. Rev. 115, 485 (1959).

[2] M. V. Berry, Proc. R. Soc. Lond. A 392, 45 (1984).

[3] N. Byers and C. N. Yang, Phys. Rev. Lett. 7, 46 (1961).

[4] F. Bloch, Phys. Rev. Lett. 21, 1241 (1968).

[5] M. Büttiker, Y. Imry, and R. Landauer, Phys. Lett. 96A, 365 (1983).

[6] For a review on the subject, see M. Peshkin and A. Tonomura, "The Aharonov-Bohm effect", in Lecture Notes in Physics 340 (Springer-Verlag, Berlin 1989).

[7] A. Tonomura, N. Osakabe, T. Matsuda, T. Kawasaki, J. Endo, S. Yano, and H. Yamada, Phys. Rev. Lett. 56, 792 (1986).

[8] R. A. Webb, S. Washburn, C. P. Umbach, and R. B. Laibowitz, Phys. Rev. Lett. 54, 2696 (1985); S. Washburn and R. A. Webb, Rep. Prog. Phys. 55, 1311 (1992).

[9] R. D. Parks and W. A. Little, Phys. Rev. 133, A97 (1964).

[10] G. Timp, A. M. Chang, J. E. Cunningham, T. Y. Chang, P. Mankiewich, R. Behringer, and R. E. Howard, Phys. Rev. Lett. 58, 2814 (1987).

[11] C. J. B. Ford, T. J. Thornton, R. Newbury, M. Pepper, H. Ahmed, C. T. Foxon, J. J. Harris, and C. Roberts, J. Phys. C: Solid State Phys., 21, L325 (1988).

[12] A. Fuhrer, S. Lüscher, T. Ihn, T. Heinzel, K. Ensslin, W. Wegscheider, and M. Bichler, Nature 413, 822 (2001); ibid., Microelectr. Eng. 63, 47 (2002).

[13] J.-B. Yau, E. P. De Poortere, and M. Shayegan, Phys. Rev. Lett. 88, 146801 (2002).

[14] B. L. Al'tshuler, A. G. Aronov, and B. Z. Spivak, Pis'ma Zh. Éksp. Teor. Fiz. 33, 101 (1981) [JETP Lett. 33, 94 (1981)].

[15] M. Büttiker, Y. Imry, R. Landauer, and S. Pinhas, Phys. Rev. B 31, 6207 (1985).

[16] A. G. Aronov and Yu. V. Sharvin, Rev. Mod. Phys. 59, 755 (1987).

[17] A. Lorke, R. J. Luyken, A. O. Govorov, J. P. Kotthaus, J. M. Garcia, and P. M. Petroff, Phys. 
Rev. Lett. 84, 2223 (2000).

[18] A. V. Chaplik, Pis'ma Zh. Éksp. Teor. Fiz. 62, 885 (1995) [JETP Lett. 62, 900 (1995)].

[19] A. B. Kalameǐtsev, V. M. Kovalev, and A. O. Govorov, Pis'ma Zh. Éksp. Teor. Fiz. 68, 634 (1998) [JETP Lett. 68, 669 (1998)].

[20] R. A. Römer and M. E. Raikh, Phys. Rev. B 62, 7045 (2000).

[21] J. Song and S. E. Ulloa, Phys. Rev. B 63, 125302 (2001).

[22] H. Hu, J.-L. Zhu, D.-J. Li, and J.-J Xiong, Phys. Rev. B 63, 195307 (2001).

[23] A. O. Govorov, A. V. Kalameǐtsev, R. Warburton, K. Karrai, and S. E. Ulloa, Physica E 13, 297 (2002).

[24] A. O. Govorov, S. E. Ulloa, K. Karrai, and R. J. Warburton, Phys. Rev. B 66, 081309 (2002);

S. E. Ulloa, A. O. Govorov, A. V. Kalameǐtsev, R. Warburton, and K. Karrai, Physica E 12, 790 (2002); A. V.Maslov and D. S. Citrin, Phys. Rev. B 67, 121304 (2003).

[25] M. Bayer, M. Korkusinski, P. Hawrylak, T. Gutbrod, M. Michel, and A. Forchel, Phys. Rev. Lett. 90, 186801 (2003).

[26] K. L. Janssens, B. Partoens, and F. M. Peeters, Phys. Rev. B 64, 155324 (2001).

[27] K. L. Janssens, B. Partoens, and F. M. Peeters, Phys. Rev. B 66, 075314 (2002).

[28] F. Hatami, N. N. Ledentsov, M. Grundmann, J. Bhrer, F. Heinrichsdorff, M. Beer, D. Bimberg, S. S. Ruvimov, P. Werner, U. Gösele, J. Heydenreich, U. Richter, S. V. Ivanov, B. Ya. Meltser, P. S. Kop'ev, and Zh. I. Alferov, Appl. Phys. Lett. 67, 656 (1995).

[29] P. Schittenhelm, M. Gail, J. Brunner, J. F. Nützel, and G. Abstreiter, Appl. Phys. Lett. 67, 1292 (1995); A. I. Yakimov, N. P. Stepina, A. V. Dvurechenskii, A. I. Nikiforov, and A. V. Nenashev, Phys. Rev. B 63, 045312 (2001).

[30] M. Hayne, R. Provoost, M. K. Zundel, Y. M. Manz, K. Eberl, and V. V. Moshchalkov, Phys. Rev. B 62, 10324 (2000); M. Sugisaki, H.-W. Hen, S. V. Nair, K. Nishi, and Y. Masumoto, Phys. Rev. B 66, 235309 (2002).

[31] E. Ribeiro, R. L. Maltez, W. Carvalho Jr., D. Ugarte, and G. Medeiros-Ribeiro, Appl. Phys. Lett. 81, 2953 (2002).

[32] Z. Xu and P. M. Petroff, J. Appl. Phys. 69, 6564 (1991).

[33] R. L. Maltez, E. Ribeiro, W. Carvalho Jr., D. Ugarte, and G. Medeiros-Ribeiro, J. Appl. Phys. 94, 3051 (2003).

[34] M. K. K. Nakaema, F. Iikawa, M. J. S. P. Brasil, E. Ribeiro, G. Medeiros-Ribeiro, W. Carvalho 
Jr., M. Z. Maialle, and M. H. Degani, Appl. Phys. Lett. 81, 2743 (2002).

[35] P. D. Wang, J. L. Merz, S. Fafard, R. Leon, D. Leonard, G. Medeiros-Ribeiro, M. Oestreich, P. M. Petroff, K. Uchida, N. Miura, H. Akiyama, and H. Sakaki, Phys. Rev. B 53, 16458 (1996); L. R. Wilson, D. J. Mowbray, M. S. Skolnick, M. Morifuji, M. J. Steer, I. A. Larkin, and M. Hopkinson, Phys. Rev. B 57, 2073 (1998); M. Hayne, J. Maes, V. V. Moshchalkov, Y. M. Manz, O. G. Schmidt, and K. Eberl, Appl. Phys. Lett. 79, 45 (2001).

[36] C. C. B. Bufon et al., manuscript in preparation.

[37] V. Fock, Z. Phys. 47, 446 (1928). 